\title{
SINGULAR BOUNDARY VALUE PROBLEMS FOR QUASI-DIFFERENTIAL EQUATIONS
}

\section{PAUL W. ELOE}

Department of Mathematics

University of I)ayton

Dayton, Ohio 15169) ISA

\section{JOHNNY HENDERSON}

('enter for 1)rnamical Systems and Nonlinear Studies (icorgia Institute of Technology, Atlanta, Georgia 30:332 and

Department of Discrete and Statistical Sciences

Auburn Iniversity, Auburn, Alabama 36849 USA

(Received April 13, 1993)

\begin{abstract}
Solutions are obtained of boundary value problems for $L_{n} y+$ $\int\left(x, L_{0} y, \ldots, L_{n-2} y\right)$, satisfying $L_{2} y(0)=L_{n-1} y(1)=0,0 \leq i \leq n-2$, where $L_{2}$ denotes the $i^{t h}$ (quasiderivative, and where $f\left(x, y_{1}, \ldots, y_{n-1}\right)$ has singularities at $y_{2}=0,1 \leq i \leq n-1$.
\end{abstract}

KEYWORDS AND PIIRASES. Quasi-derivative, singular boundary value problem, cone.

1992 AMS CLASSIFICATION CODE. 34B15.

\section{INTRODUCTION.}

We first define the quasiderivative operators, $L_{i}, 0 \leq i \leq n$, inductively by,

$$
\begin{aligned}
& L_{0} u=p_{0} u, \\
& L_{\imath} u=p_{\imath}\left(L_{\imath-1} u\right)^{\prime}, \quad 1 \leq i \leq n,
\end{aligned}
$$

where $p_{\imath}(x) \in C^{(n-\imath)}([0,1],(0, \infty)), 0 \leq i \leq n$, and we assume $p_{n-1}(x) \equiv 1$ on $[0,1]$. We will be concerned with solutions of boundary value problems for the quasi-differential equation,

$$
L_{n} y+f\left(x, L_{0} y, L_{1} y, \ldots, L_{n-2} y\right)=0 .
$$

satisfying the boundary conditions,

$$
L_{\imath} y(0)=0,0 \leq i \leq n-2 \text {, and } L_{n-1} y(1)=0,
$$

where $f\left(x, y_{1}, \ldots, y_{n-1}\right)=0$ has singularities at $y_{2}=0,1 \leq i \leq n-1$. For notation, we let

$$
\delta_{\imath}=\inf _{0 \leq x \leq 1} p_{i}(x), 0 \leq i \leq n,
$$

and

$$
\Delta_{\imath}=\sup _{0 \leq x \leq 1} p_{\imath}(x), 0 \leq i \leq n
$$

and we assume throughout that, 
(ג) $f\left(x, y_{1}, \ldots y_{n-1}\right):(0,1) \times(0 . x)^{n-1}-(0, x)$ is continuous;

(B) $f\left(x, y_{1}, \ldots, y_{n-1}\right)$ is decredsing in $y_{2}$. for each fixed $\left(x, y_{1} \ldots, y_{t-1}, y_{t+1}\right.$. $\left.\ldots, y_{n}\right), 1 \leq 1 \leq n-1$;

((') $\int_{0}^{1} f\left(x, y_{1}, \ldots, y_{n-1}\right) d x<x$. for each fixed $\left(y_{1}, \ldots, y_{n-1}\right)$;

(1)) $\lim _{y_{1} \rightarrow+0} f\left(x, y_{1} \ldots \ldots y_{n-1}\right)=+x$ uniformly on compact subsets of $(0,1) \times(0, \infty)^{n-2}, 1 \leq 1 \leq n-1$;

(l) $\lim _{y_{1} \rightarrow+\infty} f\left(x, y_{1} \ldots \ldots y_{n-1}\right)=0$ uniformly on compact subsets of $(0.1) \times(0, \infty)^{n-2} .1 \leq 1 \leq n-1$.

We observe that, if $y$ is a solution of (1.1), then by $(A), L_{n} y<0$, so that $L_{n-2} y$ is a concave function.

The singular boundary value problem (1.1), (1.2) generalizes in some sense the second order nonlinear singular problems considered by Bobisud [1], Bobisud and Lee [2], Do and Lee [3], Garner and Shivaji [4], O'Regan [5], Tineo [6], and Wang [7]-[8]. Among those works, [1], [4], and [8] used singular boundary value problems to model diffusion problems arising in physiology and physics, while in [5]. singular problems included as special cases the Thomas-Fermi and Emden-Fowler equations. Morcover, in [1], [2], [3], [5], [6], and [7] - [8], a proor bounds are established on solutions, and then Schauder degree or Granas topological transversality applications yield solutions of the singular boundary value problems. Others have also used these methods (in the case $p_{z}(x) \equiv 1,0 \leq i \leq n$, so that $L_{n}=\frac{d^{n}}{d t^{n}}$ ), for singular boundary value problems, with the paper by Eloe and Henderson [9] containing many references to those works.

Our motivation for the techniques used in obtaining solutions of (1.1), (1.2) are the works by [10] and [11], followed by the papers by Eloe and Henderson [12] and Henderson and Yin [13]. These arguments involve concavity properties, an iterative technique, and a fixed point theorem due to [11] for mappings that are decreasing with respect to a cone in a Banach space. In Section 2, we state some properties of a cone in a Banach space, followed by the fixed point theorem. In Section 3, we construct a suitable cone and define a sequence of modifications of $f$, so that none of these modifications have the singularities of $f$. For this sequence, we construct a sequence of operators, each of which satisfies the hypotheses of the fixed point theorem, hence obtaining a sequence of iterates in the cone. The sequence is shown to converge to a solution of $(1.1),(1.2)$ in the cone.

\section{SOME PRELIMINARIES.}

The following definitions and properties of cones in a Banach space can be found in Amann's [14] treatise.

Let $\mathcal{B}$ be a Banach space, and $H^{\prime}$ a closed, nonempty subset of $\mathcal{B}$. $K$ is a cone provided (i) $\alpha u+\beta v \in K$, for all $u, v \in K$ and all $\alpha, \beta \geq 0$, and (ii) $u,-u \in K$ imply $u=0$. Given a cone $K$, a partial order, $\leq$, is induced on $\mathcal{B}$ by $x \leq y$, for $x, y \in \mathcal{B}$, iff $y-x \in K$. (We may sometimes write $x \leq y$ (wrt K).) If $x, y \in \mathcal{B}$ with $x \leq y$, let $\langle x, y\rangle$ denote the closed order interval between $x$ and $y$ given by $\langle x, y\rangle=\{z \in \mathcal{B} \mid x \leq z \leq y\}$. A cone $K$ is normal in $\mathcal{B}$ provided there exists a $\delta>0$ such that $\left\|e_{1}+e_{2}\right\| \geq \delta$, for all $\epsilon_{1}, \epsilon_{2} \in K$ with $\left\|e_{1}\right\|=\left\|e_{2}\right\|=1$. 
We remark that. if $h$ is a normal cone in $\mathcal{B}$. then closed order intervals are norm bounded.

We now state a fixed point theorem due to Ciatica. Oliker, and Waltman [11] for operators that are decreasing with respect to a cone.

THEOREM 1. Lr $\mathcal{B}$ ix a Banach space. $K$ a normal cons in $\mathcal{B}, E \subseteq K$ such that. if $x, y \in E$ wath $x \leq y$, then $\langle x, y\rangle \subseteq E$, and lot $T: E-K$ be a contunuous mapping that is derreasing woth respert to $K$, and wheh is compart on any rlosed order interral contanncd in E. Suppose there exists $x_{0} \in E$ such that $T^{2} x_{0}=T\left(T x_{0}\right)$ is de fincd. and both $T x_{0}$ and $T^{2} x_{0}$ arr order comparable to $x_{0}$. If either. (2) $T x_{0} \leq x_{0}$ and $T^{2} x_{0} \leq x_{0}$ or (ul) $x_{0} \leq T x_{0}$ and $x_{0} \leq T^{2} x_{0}$, then $T$ has fixed point in $E$.

\section{SOLUTIONS OF (1.1), (1.2).}

In this section, we will apply Theorem 1 to a sequence of operators that are decreasing with respect to an appropriate cone. We then obtain a sequence of iterates from these fixed points which converges to a solution of $(1.1),(1.2)$. Concavity of the $(n-2)^{n d}$ quasiderivative of a solution plays a role in this construction.

Let the Banach space $\mathcal{B}=C^{(n-2)}[0,1]$ with norm

$$
\|y\|=\max \left\{\left|L_{0} y\right|_{\infty}, \ldots,\left|L_{n-2} y\right|_{\infty}\right\}
$$

where $|\cdot|_{\infty}$ denotes the supremum norm, and let

$$
K=\left\{y \in \mathcal{B} \mid L_{\imath} y(x) \geq 0 \text { on }[0,1], 0 \leq i \leq n-2\right\}
$$

$K$ is a normal cone in $\mathcal{B}$. We also note that, if $u, v \in \mathcal{B}$ and $u \leq v$ (wrt $K^{*}$ ), then

$$
L_{i} u(x) \leq L_{i} v(x) \text { on }[0,1], 0 \leq i \leq n-2 .
$$

In addition, we will have need of the sign of the Green's function, $G(x, s)$, for the problem,

$$
-L_{n} y=0, L_{\imath} y(0)=0,0 \leq i \leq n-2, \text { and } L_{n-1} y(1)=0
$$

Eloe [15] has proved

$$
\left(L_{\imath}\right)_{x} G(x, s)>0 \text { on }(0,1) \times(0,1), 0 \leq i \leq n-2
$$

By a solution, $y$, of $(1.1),(1.2)$, we mean $y \in C^{(n)}(0,1) \cap C^{(n-1)}[0,1], y$ satisfies $(1.1)$ on $(0,1), y$ satisfies (1.2), and $L_{\imath} y(x)>0$ on $(0,1), 0 \leq i \leq n-2$. For such, we seek a fixed point of the integral operator,

$$
T \varphi(x)=\int_{0}^{1} G(x, s) f\left(s, L_{0} \varphi(s), \ldots, L_{n-2} \varphi(s)\right) d s .
$$

But because of the singularities in $f$ given by (D), we cannot define $T$ on all of the cone $K$. We next let $g_{1}:[0,1] \rightarrow[0, \infty)$ be the solution of

$$
L_{n} u=0
$$




$$
\begin{gathered}
L_{a} u(0)=0.0 \leq 1 \leq n-2, \\
I_{n-1} u(0)=1 .
\end{gathered}
$$

and. for each $\theta>0$, define

$$
g_{H}(x)=\theta g_{1}(x) .
$$

Wo note that, for $\theta>0$, and $0 \leq 1 \leq n-2$.

$$
\begin{gathered}
L_{\imath} g_{\theta}(x)= \\
\theta \int_{0}^{x} \int_{0}^{\tau_{n-1-1}} \cdots \int_{0}^{\tau_{2}} \frac{1}{p_{1+1}\left(\tau_{n-1-1}\right) \cdots p_{n-2}\left(\tau_{2}\right) p_{n-1}\left(\tau_{1}\right)} d \tau_{1} d \tau_{2} \cdots d \tau_{n-1-1},
\end{gathered}
$$

and

$$
L_{n-1} g_{\theta}(x) \equiv \theta \text { on }[0,1]
$$

so that $g_{\theta} \in K$, and in fact $L_{2} g_{\theta}(x)>0$ on $(0,1], 0 \leq 1 \leq n-2$.

Assume for the remainder of the paper.

(F) For each $\theta>0 . \int_{0}^{1} f\left(x, L_{0} g_{\theta}(x), L_{1} g_{\theta}(x), \ldots L_{n-2} g_{\theta}(x)\right) d x<\infty$.

Finally, we define $D \subseteq K$ by

$$
D=\left\{\varphi \in \mathcal{B} \mid \text { there exits } \theta(\varphi)>0 \text { such that } g_{\theta} \leq \varphi\left(\text { wrt } K^{*}\right)\right\} \text {, }
$$

and define $T: D \rightarrow K$ by

$$
T \varphi(x)=\int_{0}^{1}\left(i(x, s) \int\left(s, L_{0} \varphi(s), L_{1} \varphi(s), \ldots, L_{n-2} \varphi(s)\right) d s, 0 \leq x \leq 1, \varphi \in D .\right.
$$

It follows from (A) - (F) and properties of $G(x, s)$ that, if $\varphi \in D$, then $T \varphi \in C^{(n)}(0,1) \cap C^{(n-1)}[0,1], T \varphi$ satisfies $(1.2), L_{t}(T \varphi)(x)>0$ and increasing on $(0,1], 0 \leq i \leq n-2, L_{n-2}(T \varphi)$ is concave on [0,1], and that $T \varphi \in D$. On the other hand, if $\varphi \in C^{(n)}(0,1) \cap C^{(n-1)}[0,1]$ is a solution of (1.1), (1.2), with $L_{\imath} \varphi(x)>0$ on $(0,1], 0 \leq i \leq n-2$, it again follows from the concavity of $L_{n-2} \varphi$ that $\varphi \in D$. Consequently, $\varphi \in D$ is a solution of (1.1), (1.2) iff $T \varphi=\varphi$.

Our first result of this section gives a priori bounds on $L_{n-2} \varphi$, for all solutions $\varphi$ of (1.1), (1.2), that belong to $D$.

THEOREM 2. Assume that $(A)-(F)$ are satisfied. Then, there exists an $R>0$ such that $\left|L_{n-2} \varphi\right|_{\infty} \leq$ $R$, for all solutions $\varphi$ of (1.1), (1.2), that belong to $D$.

PROOF. Assume the conclusion to be false. Then, there is a sequence $\left\{\varphi_{\ell}\right\} \subseteq D$ of solutions of (1.1), (1.2). such that $\lim _{\ell \rightarrow \infty}\left|I_{n-2} \varphi_{\ell}\right|_{\infty}=\infty$. We may assume that, for each $\ell \geq 1$,

$$
\left|L_{n-2} \varphi_{\ell}\right|_{\infty} \leq\left|L_{n-2} \varphi_{\ell+1}\right|_{\infty}
$$

From the equation (1.1), $L_{n-1} \varphi_{\ell}(x)>0$ and decreasing on $[0,1)$, and from (1.2), $L_{\imath} \varphi_{\ell}(x)>0$ and increasing on $(0.1], 0 \leq i \leq n-2$. It follows that, for each $\ell$,

$$
0 \leq L_{n-2} \varphi_{\ell}(1)=\left|L_{n-2} \varphi_{\ell}\right|_{\infty} \leq\left|L_{n-2} \varphi_{\ell+1}\right|_{\infty}=L_{n-2} \varphi_{\ell+1}(1)
$$




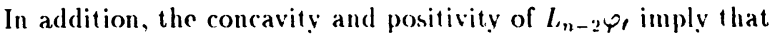

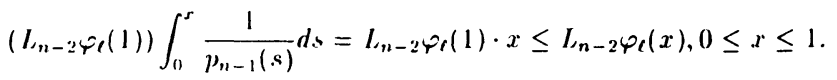

So, from the monotonicity in (3.5) and (3.6), if we set $\theta=L_{n-2} \varphi_{1}(1)$, then

$$
L_{n-2 !} g_{\theta}(x) \leq L_{n-2} \varphi_{\ell}(x) \text { on }[0,1], \ell \geq 1
$$

From the conditions satisfied by $g_{\theta}$ and $\varphi_{\ell}$ at $x=0$, upon multiplying successively by $\left(p_{2}(x)\right)^{-1}$ and integrating, we obtain

$$
g_{\theta} \leq \varphi_{\ell}\left(\text { wrt } K^{*}\right), \text { for all } \ell \geq 1 \text {. }
$$

Now, set

$$
0<M=\sup _{[0,1] \times[0,1]}\left(L_{n-2}\right)_{x} G(x, s) .
$$

Then (B) and (F) yield that, for $0 \leq x \leq 1$ and $\ell \geq 1$,

$$
\begin{aligned}
L_{n-2} \varphi_{\ell}(x) & =L_{n-2}\left(T \varphi_{\ell}\right)(x) \\
& =\int_{0}^{1}\left(L_{n-2}\right)_{x} G(x, s) f\left(s, L_{0} \varphi_{\ell}(s), \ldots, L_{n-2} \varphi_{\ell}(s)\right) d s \\
& \leq M \int_{0}^{1} f\left(s, L_{0} g_{\theta}(s), \ldots, L_{n-2} g_{\theta}(s)\right) d s \\
& =N
\end{aligned}
$$

for some $0<N<\infty$. But $0 \leq x \leq 1$ and $\ell \geq 1$ were arbitrary. So

$$
\left|L_{n-2} \varphi_{\ell}\right|_{\infty} \leq N, \text { for all } \ell \geq 1
$$

which contradicts $\lim _{\ell \rightarrow \infty}\left|L_{n-2} \varphi_{\ell}\right|_{\infty}=\infty$. The proof is complete.

COROLLARY . Assume (A)-(F) are satisfied. Then there exists an $R>0$ such that $0 \leq L_{i} \varphi(x) \leq$ $\left(\frac{R}{\delta_{n-2} \cdots \delta_{i+1}}\right) \frac{x^{n-i-2}}{(n-i-2) !}$ on $[0,1]$, for $0 \leq i \leq n-2$, and $\|\varphi\| \leq \sup _{0 \leq i \leq n-2}\left\{\frac{R}{\delta_{n-2} \cdots \delta_{i+1}}\right\} \equiv \tilde{R}$, for all solutions $\varphi$ of (1.1), (1.2) that belong to $D$. In particular, $\varphi \leq \frac{\tilde{R}}{(n-2) !} x^{n-2}$ (wrt $\left.K\right)$, for all solutions $\varphi \in D$ of (1.1), (1.2).

Next, for each $\ell \geq 1$, let $\psi_{\ell}:[0,1] \rightarrow[0, \infty)$ be defined by

$$
\psi_{\ell}(x)=\int_{0}^{1} G(x, s) f(s, \ell, \ldots, \ell) d s .
$$

With assumptions (A) - (E), we have

$$
0<L_{\imath} \psi_{\ell+1}(x) \leq L_{i} \psi_{\ell}(x) \text { on }(0,1), 0 \leq i \leq n-2
$$

Furthermore,

$$
\lim _{\ell \rightarrow \infty} L_{i} \psi_{\ell}(x)=0 \text { uniformly on }[0,1], 0 \leq i \leq n-2 .
$$

Now, define a sequence of functions, $f_{\ell}\left(x, y_{1}, \ldots, y_{n-1}\right):(0,1) \times[0, \infty)^{n-1} \rightarrow(0, \infty)$ by

$$
f_{\ell}\left(x, y_{1}, \ldots, y_{n-1}\right)=f\left(x, \max \left\{y_{1}, L_{0} \psi_{\ell}(x)\right\}, \ldots, \max \left\{y_{n-1}, L_{n-2} \psi_{\ell}(x)\right\}\right)
$$


For $\ell \geq 1, f_{\ell}$ is continuous and satisfies (B). Also, from (B), we have, for each $\ell \geq 1$,

$$
f_{1}\left(x, y_{1}, \ldots, y_{n-1}\right) \leq f\left(x, y_{1}, \ldots, y_{n-1}\right) \text { on }(0,1) \times(0, \infty)^{n-1}
$$

and

$$
f_{\ell}\left(x, y_{1}, \ldots, y_{n-1}\right) \leq f\left(x, L_{0} \psi_{\ell}(x), \ldots, L_{n-2} \psi_{\ell}(x)\right) \text { on }(0,1) \times(0, \infty)^{n-1}
$$

THEOREM 3. Assume that conditions (A)-(F) are satisfied. Then the boundary value problem (1.1), (1.2) has a solution, $y$, such that $L_{\imath} y(x)>0$ on $(0,1), 0 \leq i \leq n-2$.

PROOF. We begin by defining a sequence of compact mappings $T_{\ell}: K \rightarrow K$ by

$$
T_{\ell} \varphi(x)=\int_{0}^{1} G(x, s) f_{\ell}\left(s, L_{0} \varphi(s), \ldots, L_{n-2} \varphi(s)\right) d s, 0 \leq x \leq 1, \varphi \in K .
$$

For $\ell \geq 1$ and $\varphi \in K, L_{n-2}\left(T_{\ell} \varphi\right)>0$ is concave on $(0,1), T_{\ell} \varphi$ satisfies the boundary conditions (1.2), and from $\left(L_{\imath}\right)_{x} G(x, s)>0,0 \leq i \leq n-2$, we have $L_{\imath}(T \varphi)>0$ and increasing on $(0,1), 0 \leq i \leq n-2$.

Since each $f_{\ell}$ satisfies (B), it follows that $T_{\ell}$ is decreasing with respect to the cone $K$, for each $\ell \geq 1$. Also, $0 \leq T_{\ell}(0)$ (wrt $K$ ) and $0 \leq T_{\ell}^{2}(0)$ (wrt $K$ ), and so by Theorem 1 , for each $\ell$, there exists a $\varphi_{\ell} \in K$ such that $T_{\ell} \varphi_{\ell}=\varphi_{\ell}$. From our observations above,

$$
\left|L_{n-2} \varphi_{\ell}\right|_{\infty}=L_{n-2} \varphi_{\ell}(1)
$$

By essentially the same arguments as in Theorem 2 , it follows that there is an $R>0$ such that, for each $\ell \geq 1$,

$$
\left|L_{n-2} \varphi_{\ell}\right|_{\infty} \leq R \text { and }\left\|\varphi_{\ell}\right\| \leq \tilde{R}
$$

where $\tilde{R}$ is given in the Corollary.

Our next claim is that there exists $k>0$ such that $k \leq\left|L_{n-2} \varphi_{\ell}\right|_{\infty}$, all $\ell \geq 1$. Assume the claim is false. Then passing to a subsequence and relabeling, we have without loss of generality that $\lim _{\ell \rightarrow \infty}\left|L_{n-2} \varphi_{\ell}\right|_{\infty}=0$, which implies, along with the boundary conditions (1.2),

$$
\lim _{\ell \rightarrow \infty} L_{\imath} \varphi_{\ell}(x)=0 \text { uniformly on }[0,1], 0 \leq i \leq n-2
$$

Let $0<\delta<\frac{1}{2}$ be fixed and let

$$
0<m=i n f_{[\delta, 1-\delta] \times[\delta, 1-\delta]} L_{n-2} G(x, s) .
$$

By (D), there exists $\eta>0$ such that, if $\delta \leq x \leq 1-\delta$ and $0<y_{2}<\eta$, for $1 \leq i \leq n-1$, then

$$
f\left(x, y_{1}, \ldots, y_{n-1}\right)>\frac{2}{m}
$$

From (3.7), there exits $\ell_{0} \geq 1$ such that, for $\ell \geq \ell_{0}$,

$$
0<\left|L_{\imath} \varphi_{\ell}(x)\right|<\eta / 2,0<x<1,0 \leq i \leq n-2
$$

Also, for some $\ell_{1} \geq \ell_{0}$ it follows that, if $\ell \geq \ell_{1}$,

$$
0<\left|L_{\iota} \psi_{\ell}(x)\right|<\eta / 2,0<x<1,0 \leq i \leq n-2 .
$$


So, for $\ell \geq \ell$ and $\delta \leq x \leq 1-\delta$.

$$
\begin{aligned}
L_{n-2 \varphi_{f} f}(x)= & \int_{0}^{1} L_{n-2}\left(x^{\prime}(x, s) f_{\ell}\left(s, L_{0} \varphi_{\ell}(s), \ldots, L_{n-2} \varphi_{\ell}(s)\right) d s\right. \\
& \geq \int_{\delta}^{1-\delta} G(x, s) f_{\ell}\left(s, L_{0} \varphi_{\ell}(s), \ldots, L_{n-2} \varphi_{\ell}(s)\right) d s \\
& \geq m \int_{\delta}^{1-\delta} f(s, \eta / 2, \ldots, \eta / 2) d s \\
& \geq 1 .
\end{aligned}
$$

This is a contradiction to (3.6). Thus, there is a $k>0$ such that $k \leq\left|L_{n-2} \varphi_{\ell}\right|_{\infty}$, for all $\ell$. With $\theta=\frac{k}{2}$, and applying (1.2), we obtain

$$
L_{2} g_{\theta}(x) \leq L_{2} \varphi_{\ell}(x) \text { on }[0,1], 0 \leq i \leq n-2, \ell \geq 1
$$

We have

$$
g_{\theta} \leq \varphi_{\ell} \leq \frac{\tilde{R}}{(n-2) !} x^{n-2}(\text { wrt } K), \ell \geq 1
$$

or, in particular,

$$
\left\{\varphi_{\ell}\right\} \subseteq\left\langle g_{\theta}, \frac{\tilde{R}}{(n-2) !} x^{n-2}\right\rangle \subseteq D
$$

When restricted to the closed order interval, $\left\langle g_{\theta}, \frac{\tilde{R}}{(n-2) !} x^{n-2}\right\rangle, T$ is a compact mapping. So, there is a subsequence of $\left\{T \varphi_{\ell}\right\}$, which we relabel as the original sequence, which converges to some $\varphi^{*} \in K$; that is, $\left\|T \varphi_{\ell}-\varphi^{*}\right\| \rightarrow 0$, as $\ell \rightarrow \infty$.

To complete the proof, we show that $\left\|T \varphi_{\ell}-\varphi_{\ell}\right\|-0$, as $\ell \rightarrow \infty$. With $\theta=\frac{k}{2}$, let $\epsilon>0$ be given, and let

$$
P=\max _{0 \leq 1 \leq n-2}\left\{\sup _{[0,1] \times[0,1]} L_{2} G(x, s)\right\}
$$

The integrability condition (F) and the absolute continuity of the integral imply there exists $0<\delta<1$ such that

$$
\begin{aligned}
& 2 P\left[\int_{0}^{\delta} f\left(s, L_{0} g_{\theta}(s), \ldots, L_{n-2} g_{\theta}(s)\right) d s+\right. \\
& \left.\int_{1-\delta}^{1} f\left(s, L_{0} g_{\theta}(s), \ldots, L_{n-2} g_{\theta}(s)\right) d s\right]<\epsilon .
\end{aligned}
$$

Also, there exists $\ell_{0}$ such that, for $\ell \geq \ell_{0}$,

$$
L_{\imath} \psi_{\ell}(x) \leq L_{\imath} g_{\theta}(x) \leq L_{\imath} \varphi_{\ell}(x) \text { on }[\delta, 1-\delta], 0 \leq i \leq n-2
$$

Observe also that $f_{\ell}\left(s, L_{0} \varphi_{\ell}(s), \ldots, L_{n-2} \varphi_{\ell}(s)\right)=f\left(s, L_{0} \varphi_{\ell}(s), \ldots, L_{n-2} \varphi_{\ell}(s)\right)$, for $\delta \leq s \leq 1-\delta$ and $\ell \geq \ell_{0}$. Thus, for $0 \leq i \leq n-2, \ell \geq \ell_{0}$, and $0 \leq x \leq 1$,

$$
\begin{aligned}
\left|L_{\imath}\left(T \varphi_{\ell}\right)(x)-L_{\imath} \varphi_{\ell}(x)\right| \leq & 2 P\left[\int_{0}^{\delta} f\left(s, L_{0} \varphi_{\ell}(s), \ldots, L_{n-2} \varphi_{\ell}(s)\right) d s\right. \\
& \left.+\int_{1-\delta}^{1} f\left(s, L_{0} \varphi_{\ell}(s), \ldots, L_{n-2} \varphi_{\ell}(s)\right) d s\right] \\
\leq & 2 P\left[\int_{0}^{1} f\left(s, L_{0} g_{\theta}(s), \ldots, L_{n-2} g_{\theta}(s)\right) d s\right. \\
& \left.+\int_{1-\delta}^{1} f\left(s, L_{0} g_{\theta}(s), \ldots, L_{n-2} g_{\theta}(s)\right) d s\right] \\
< & \epsilon .
\end{aligned}
$$


Therefore,

$$
\lim _{i \rightarrow \infty}\left\|T_{\varphi l}-\varphi \ell\right\|=0
$$

It follows, in turn. that $\lim _{1 \rightarrow \sim}\left\|\varphi,-\varphi^{*}\right\|=0$, so that

$$
\varphi^{*} \in\left\langle g_{\theta} \cdot \frac{\tilde{R}}{(n-2) !} x^{n-2}\right\rangle \subseteq D,
$$

and

$$
\hat{\varphi}^{*}=\lim _{\ell \rightarrow \infty} T \varphi_{\ell}=T\left(\lim _{\ell \rightarrow \infty} \varphi_{\ell}\right)=T \varphi^{*} .
$$

and the proof is complete.

\section{REFERENCES}

1. BOBISUD, L. E. Existence of solutions of some nonlinear diffusion problems, J. Math. Anal. Appl. 168 (1992), 41.3-124.

2. BOBISUD, L. E. and LEE, Y. S. Existence for a class of nonlinear singular boundary value problems, Appl. Anal. 38 (1990), 45-67.

3. DO, T. S. and LEE, H. II. Existence of solutions of singular nonlinear two-point boundary value problems, Kyungpook Math. J. 31 (1991), 113-118.

4. GARNER, J. B. and SHIVAJI, R. Existence and uniqueness of solutions of a class of singular diffusion problems, Appl. Anal. 30 (1988), 101-114.

5. O'REGAN, D. Existence theorems for certain classes of singular boundary value problems, J. Math. Anal. Appl. 168 (1992), 523-5.39.

6. TINEO, A. Existence theorems for a singular two-point Dirichlet problem, Nonlin. Anal. 19 (1992), 323-33.1.

7. WANG, H. Y. Solvability of some boundary value problems for the singular equation $\left(p(t) r(t, y) y^{\prime}\right)^{\prime} / q(t)$ $f\left(t, y, p r y^{\prime}\right)$, Chinese Ann. Math. Ser. A 12 (1991), 87-91 (Chinese).

8. WANG, H. Y. Solvability of some singular boundary value problems for the equation $\left(p(t) r(t, u) u^{\prime}\right)^{\prime} / q(t)$ $f\left(t, u, p r u^{\prime}\right)$, Nonlin. Anal. 18 (1992), 649-655.

9. ELOE, P. W. and IENDERSON J. Existence of solutions of some singular higher order boundary value problems, Zeit. Angew. Math. Mech. 72 (1992).

10. GATICA, J. A., HERNANDEZ, G. E., and WALTMAN, P. Radially symmetric solutions of a class of singular elliptic problems, Proc. Edlinburgh Math. Soc. 33 (1990), 169-180.

11. GATICA, J. A., OLIKER, V., and WALTMAN, P. Singular nonlinear boundary value problems for second-order ordinary differential equations, J. Diff. Eqs. 79 (1989), 62-78.

12. ELOE, P. W. and HENDERSON, J. Singular nonlinear boundary value problems for higher order ordinary differential equations, Nonlin. Anal. 17 (1991), 1-10.

13. IIENDERSON, J. and YIN, K. C. Singular boundary value problems, Bull. Inst. Math. Acad. Sinica 19 (1991), 229-242.

14. AMANN, H. Fixed point equations and nonlinear eigenvalue problems in ordered Banach spaces, SIAM Rev. 18 (1976), 620-709.

15. ELOE, P. W. Sign properties of Cireen's functions for two classes of boundary value problems, Canad. Math. Bull. 30 (1987), 28-35. 


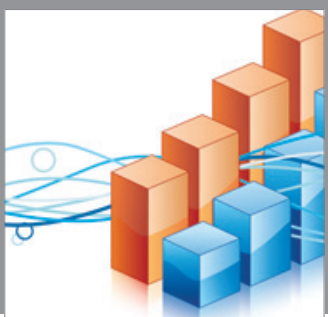

Advances in

Operations Research

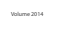

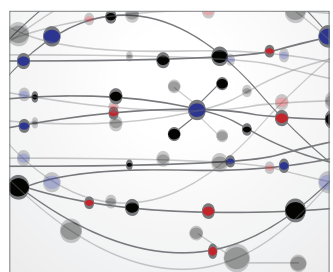

\section{The Scientific} World Journal
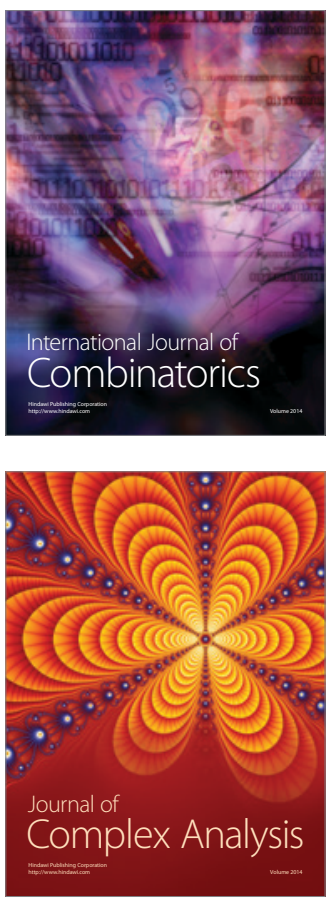

International Journal of

Mathematics and

Mathematical

Sciences
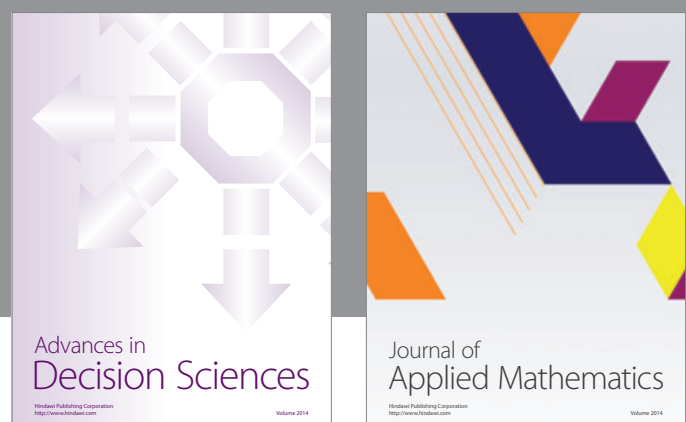

Journal of

Applied Mathematics
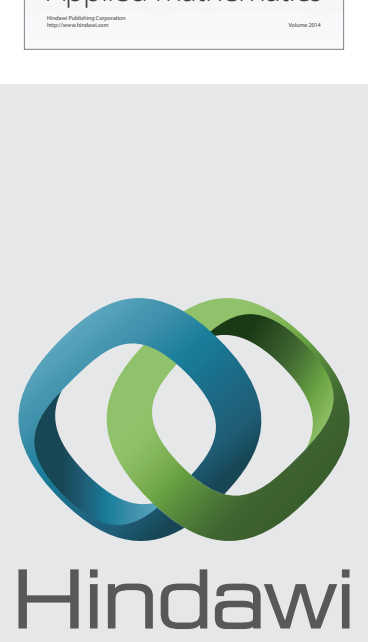

Submit your manuscripts at http://www.hindawi.com
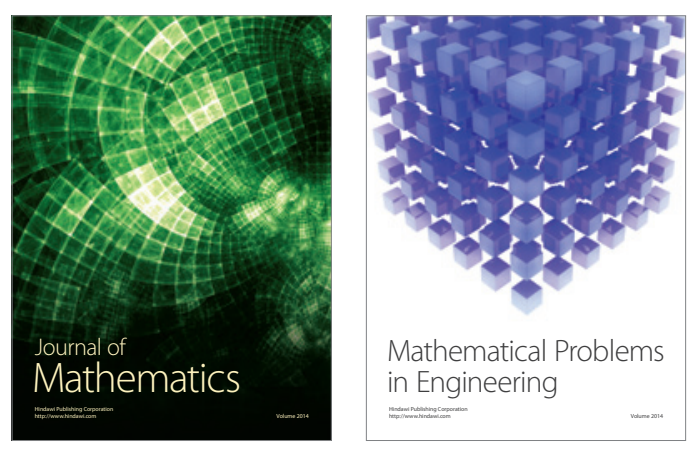

Mathematical Problems in Engineering
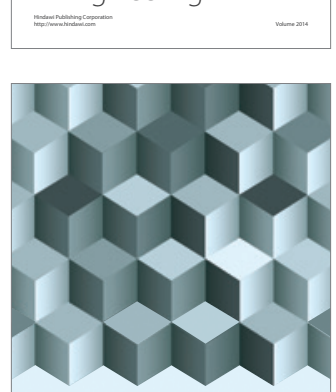

Journal of

Function Spaces
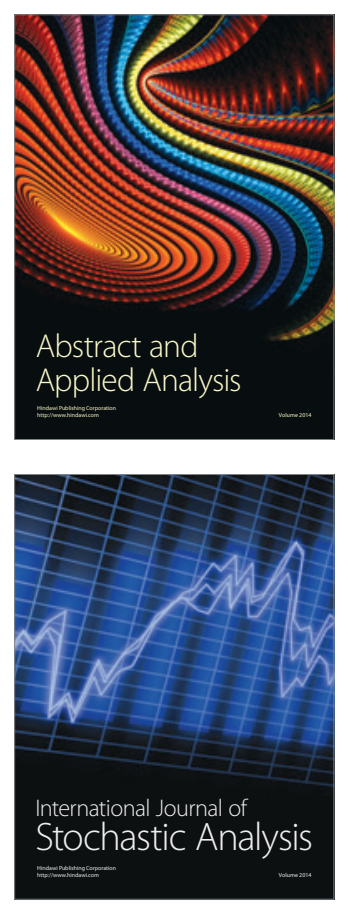

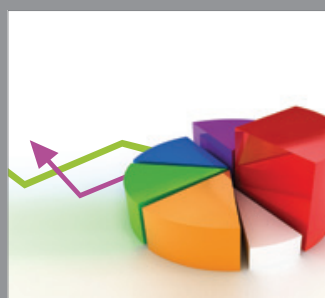

ournal of

Probability and Statistics

Promensencen
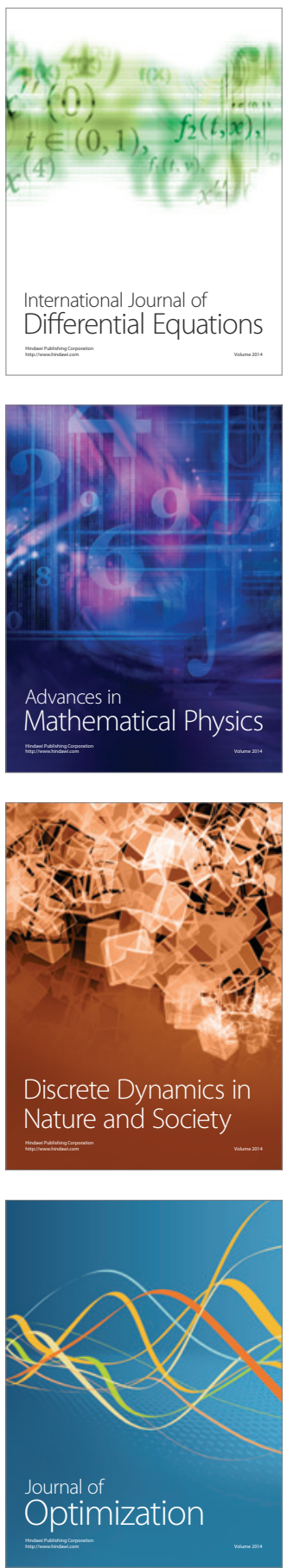